\title{
Core-shell nanofibers of curcumin/cyclodextrin inclusion complex and polylactic acid: Enhanced water solubility and slow release of curcumin
}

\author{
Zeynep Aytac ${ }^{\mathrm{a}, \mathrm{b}}$, Tamer Uyar ${ }^{\mathrm{a}, \mathrm{b}, *}$ \\ a Institute of Materials Science E Nanotechnology, Bilkent University, Ankara 06800, Turkey \\ ${ }^{\mathrm{b}}$ UNAM-National Nanotechnology Research Center, Bilkent University, Ankara 06800, Turkey
}

\section{A R T I C L E I N F O}

\section{Article history:}

Received 30 November 2016

Received in revised form 24 December 2016

Accepted 31 December 2016

Available online 3 January 2017

\section{Keywords:}

Electrospinning

Core-shell

Curcumin

Hydroxypropyl- $\beta$-cyclodextrin

Slow release

Antioxidant activity

\begin{abstract}
A B S T R A C T
Core-shell nanofibers were designed via electrospinning using inclusion complex (IC) of model hydrophobic drug (curcumin, CUR) with cyclodextrin (CD) in the core and polymer (polylactic acid, PLA) in the shell (cCUR/HP $\beta C D-I C-S P L A-N F)$. CD-IC of CUR and HP $\beta C D$ was formed at 1:2 molar ratio. The successful formation of core-shell nanofibers was revealed by TEM and CLSM images. cCUR/HP $\beta C D-I C-$ sPLA-NF released CUR slowly but much more in total than PLA-CUR-NF at pH 1 and pH 7.4 due to the restriction of CUR in the core of nanofibers and solubility improvement shown in phase solubility diagram, respectively. Improved antioxidant activity of cCUR/HP $\beta C D-I C-s P L A-N F$ in methanol:water $(1: 1)$ is related with the solubility enhancement achieved in water based system. The slow reaction of cCUR/HP $\beta C D-I C-S P L A-N F$ in methanol is associated with the shell inhibiting the quick release of CUR. On the other hand, cCUR/HP $\beta C D-I C-s P L A-N F$ exhibited slightly higher rate of antioxidant activity than PLACUR-NF in methanol:water (1:1) owing to the enhanced solubility. To conclude, slow release of CUR was achieved by core-shell nanofiber structure and inclusion complexation of CUR with HP $\beta C D$ provides high solubility. Briefly, electrospinning of core-shell nanofibers with CD-IC core could offer slow release of drugs as well as solubility enhancement for hydrophobic drugs.
\end{abstract}

(c) 2017 Elsevier B.V. All rights reserved.

\section{Introduction}

Curcumin (CUR) (Fig. 1a) is a polyphenol and apart from its usage as a therapeutic agent, it is widely employed as a spice, food preservative, flavoring and coloring agent (Aggarwal et al., 2003). Its common application for various diseases including cancer, cardiovascular and Alzheimer's disease, inflammatory and neurological disorders is owing to the outstanding biological functions such as antioxidant, anti-tumor, and anti-inflammatory activities of CUR (Yallapu et al., 2015). But, it also exhibits drawbacks like low bioavailability, instability depending on $\mathrm{pH}$, insolubility in water, slow uptake by the cells and rapid metabolism inside the cell (Siviero et al., 2015). Several strategies were developed previously to improve pharmacokinetics, systemic bioavailability, and biological activity of CUR (Siviero et al., 2015). Among these strategies, cyclodextrin (CD) inclusion complexes (ICs) is a commonly applied method to overcome the limitations of CUR. CDs are nontoxic and biodegradable cyclic oligosaccharides which are capable of

\footnotetext{
* Corresponding author at: Institute of Materials Science \& Nanotechnology, Bilkent University, Ankara 06800, Turkey.

E-mail addresses: tamer@unam.bilkent.edu.tr, tameruyar@gmail.com (T. Uyar).
}

forming ICs with a variety of molecules to enhance solubility, bioavailability, and thermal stability of hydrophobic guest compounds; reduce the volatility of molecules with low thermal stability, mask off malodors/bitter tastes, and control release of active agents (Del Valle, 2004; Hedges 1998; Szejtli, 1998). The most common CDs are $\alpha-C D, \beta-C D$ and $\gamma-C D$ with 6,7 , and 8 glucose units, respectively. In addition, chemically modified CDs including hydroxypropyl-beta-cyclodextrin (HPßCD) (Fig. 1b) in which some of the hydroxyl groups in the $\beta-C D$ structure are substituted with hydroxypropyl groups were also synthesized. $\mathrm{HP} \beta C D$ is more suitable for the solubilization of hydrophobic drugs due to its better aqueous solubility compared to native $\beta-C D$ (Del Valle, 2004; Hedges 1998; Szejtli, 1998). IC of CUR and HP $\beta C D$ were studied before for several aims such as enhancing the solubility and fluorescence (Baglole et al., 2005), oral bioavailability (Bansal et al., 2011) of CUR; treating melanoma (Sun et al., 2014), and inflammatory bowel disease (Yadav et al., 2009).

Nanofibers are quite appropriate to carry active agents including drugs, antioxidant, and antibacterial agents owing to high surface to volume ratio and porous structure (Agarwal et al., 2008). Furthermore, owing to the morphological similarities of nanofibers with extracellular matrix, biomaterials for wound healing and scaffolds for tissue engineering could be developed by 
a.

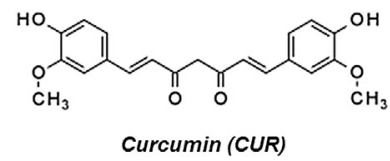

Curcumin (CUR) b.

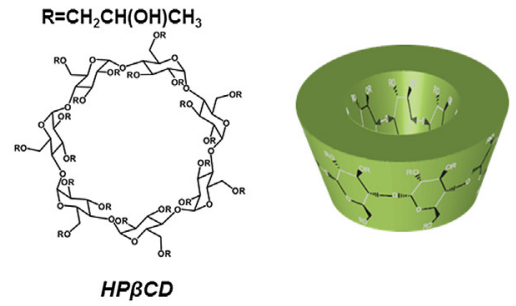

d.

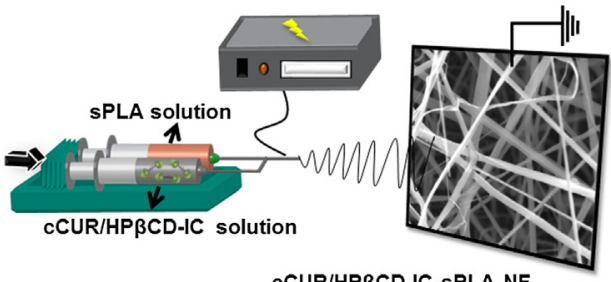

C.

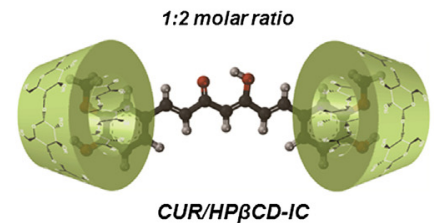

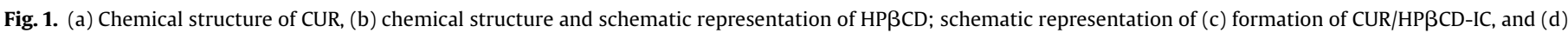
electrospinning of core-shell nanofibers from cCUR/HPßCD-IC-sPLA solution.

using nanofibers (Greiner and Wendorff, 2007; Wendorff et al., 2012). Recently, there has been significant interest on electrospinning which is a simple and common technique for producing nanofibers (Greiner and Wendorff, 2007; Wendorff et al., 2012). Design flexibility of electrospun nanofibers facilitates the encapsulation of active agents for biomedical applications (Greiner and Wendorff, 2007; Wendorff et al., 2012). CUR loaded electrospun nanofibers were reported previously in the literature (Guo et al., 2011; Sampath et al., 2014; Suwantong et al., 2007). However, rather than loading free active agents into electrospun nanofibers, incorporating their CD-ICs is advantageous in many aspects as previously reported in the studies of our group. For instance, volatile molecules were highly preserved (Aytac et al., 2014; Kayaci et al., 2013a, 2014; Kayaci and Uyar, 2012; Uyar et al., 2009a, 2009b, 2011 ) and the solubility of hydrophobic molecules were improved (Aytac et al., 2015, 2016a, 2016b; Aytac and Uyar, 2016; Kayaci et al., 2013b) by CD-IC incorporated nanofibers. Sun et al. (2013) published a study concerning CUR/CD-IC loaded electrospun nanofibers (Sun et al., 2013). Faster release was seen from CUR/ HP $\beta C D$-IC incorporated polyvinyl alcohol (PVA) nanofibers than CUR incorporated PVA nanofibers owing to the solubility enhancement and it is expected for CUR/HPßCD-IC incorporated PVA nanofibers to exhibit higher systemic bioavailability and enhanced in vivo efficacy. On the other hand, it is of great importance for some compounds to be protected against organic solvents, encapsulated in large amount and released in a more controlled manner. Due to the flexibility of the set-up, nanofibers with different morphologies such as core-shell, aligned and hollow nanofibers can be obtained via electrospinning (Ramakrishna et al., 2005). Particularly, electrospinning of core-shell nanofibers has several advantages such as possibility to electrospun nanofibers from non-spinnable solutions (Sun et al., 2003), protecting sensitive active agents against harsh environment of organic solvents (Jiang et al., 2014), controlling the release of active agents in a more efficient way due to the presence of shell acting as an additional layer (Jiang et al., 2005), encapsulating more than one drug at the same time (Llorens et al., 2015), designing active agent containing nanofibers for targeted release (Wang et al., 2015). In the study of Llorens et al. (2015), triclosan loaded poly(ethylene glycol) and CUR loaded poly(butylene succinate) solutions were used as core or shell solutions at different compositions. The release of triclosan and CUR were investigated in PBS and PBS/ ethanol $(30: 70, v / v)$. CUR could not be released in PBS from all compositions because of its high hydrophobicity and interaction with poly(butylene succinate); whereas it was completely released in PBS/ethanol (30:70, v/v) (Llorens et al., 2015). Kumar et al. (2014) produced core-shell nanofibers by encapsulating CUR and 5 -fluorouracil in the core and then, both core and shell polymers was crosslinked in type I nanofibers; whereas only shell was crosslinked in type II nanofibers. But, crosslinking of core and shell or only shell did not affect the release rate and amount of CUR in contrast to 5-fluorouracil (Kumar et al., 2014). In the study of Sedghi and Shaabani (2016) core-shell polymer-free core structure nanofibers was produced by using CUR solution in the core and PVA and chitosan in the shell. Although the burst release of CUR was prevented by core-shell nanofibers compared to blend nanofibers, core-shell nanofibers released less amount of CUR than blend nanofibers due to the low solubility of CUR in aqueous solutions (Sedghi and Shaabani, 2016).

In this study, core-shell nanofibers of CUR/HP $\beta C D-I C$ (as a core) (Fig. 1c) and polylactic acid (PLA) (as a shell) which is an aliphatic polyester and widely used in biological applications due to its biodegradability and biocompatibility was produced via electrospinning (cCUR/HPßCD-IC-sPLA-NF) (Fig. 1d). As a control sample, CUR blended with PLA was also electrospun into nanofibers (PLACUR-NF). The molar ratio of the CUR:HP $\beta C D$ inclusion complex was $1: 2$ and the phase solubility test confirmed the water solubility increase of CUR with the inclusion complexation. Core-shell morphology of cCUR/HPßCD-IC-sPLA-NF was confirmed by TEM and CLSM imaging. In vitro release of CUR from PLA-CUR-NF and cCUR/HPßCD-IC-sPLA-NF was tested in $0.1 \mathrm{~N} \mathrm{HCl}(\mathrm{pH} 1), \mathrm{PBS}(\mathrm{pH}$ 7.4), methanol, and methanol:water (1:1). The antioxidant activity of nanofibers was investigated by 2,2-diphenyl-1-picrylhydrazyl $(\mathrm{DPPH})$ radical scavenging assay with respect to concentration and time.

\section{Experimental}

\subsection{Materials}

Polylactic acid (PLA) (Natureworks, product code 6252D) and hydroxypropyl-beta-cyclodextrin (HP $\beta C D$ ) (Wacker Chemie AG, Germany) was donated to our research group for laboratory studies. Curcumin (CUR, $\geq 95 \%$, Alfa Aesar), zinc acetate dehydrate (Sigma Aldrich), fluorescein isothiocyanate (FITC, Sigma Aldrich), potassium phosphate monobasic (Sigma Aldrich), sodium 
phosphate dibasic heptahydrate (Sigma Aldrich), sodium chloride (Sigma Aldrich), methanol (extra pure, Sigma Aldrich), chloroform $\left(\mathrm{CHCl}_{3}\right.$, extra pure, Sigma Aldrich), deuterated dimethylsulfoxide (DMSO-d6, deuteration degree min 99.8\% for NMR spectroscopy, Merck), hydrochloric acid ( $\mathrm{HCl}, 36.5-38 \%$, Sigma-Riedel), 2,2diphenyl-1-picrylhydrazyl (DPPH, Sigma Aldrich) were purchased and used as-received without any further purification. Distilleddeionized water was supplied from Millipore milli-Q ultrapure water system.

\subsection{Preparation of solutions for electrospinning}

Core-shell nanofibers of CUR/HP $\beta C D$-inclusion complex (CUR/ $\mathrm{HP} \beta C D-I C)$ as a core and PLA as a shell were produced via electrospinning (cCUR/HP $\beta C D-I C-s P L A-N F)$. As control samples, pristine PLA nanofibers (PLA-NF) and CUR blended with PLA was also electrospun (PLA-CUR-NF). PLA solution was prepared by dissolving PLA (15\%, w/v) in $\mathrm{CHCl}_{3}$ :Methanol (2:1) for $3 \mathrm{~h}$. In order to produce PLA-CUR-NF, PLA-CUR solution was prepared by dissolving 3.33\% CUR (w/w, with respect to polymer) in $\mathrm{CHCl}_{3}$ : Methanol (2:1), then PLA (15\%,w/v) was added into the solution. The solution was stirred at room temperature (RT) for $3 \mathrm{~h}$ prior to electrospinning. For core-shell nanofibers, core solution was prepared by dissolving HP $\beta C D$ in water and then adding CUR (CUR:HP $\beta C D, 1: 2$ molar ratio) and the core solution was stirred overnight at RT. In addition, PLA (15\%, w/v) was dissolved in $\mathrm{CHCl}_{3}$ : Methanol $(2: 1)$ for $3 \mathrm{~h}$ at RT to be used as shell solution. The compositions of the solutions used for the electrospinning of nanofibers are summarized in Table S1.

\subsection{Electrospinning}

PLA-NF and PLA-CUR-NF was produced by single-nozzle electrospinning. PLA and PLA-CUR solutions were loaded separately in a plastic syringe (inner diameter: $0.8 \mathrm{~mm}$ ) and mounted on a syringe pump (WPI, SP 101IZ). Then, the solutions were fed at a rate of $1 \mathrm{~mL} / \mathrm{h}$ and meanwhile $15 \mathrm{kV}$ was applied from a high voltage power supply (AU Series, Matsusada Precision Inc.). Nanofibers were deposited on a grounded cylindrical metal covered with aluminum foil at a distance of $10 \mathrm{~cm}$ from the needle tip. In order to electrospun cCUR/HP $\beta C D$-IC-sPLA-NF, home-made core-shell setup was used (Fig. 1d). Core and shell solutions were loaded in plastic syringes mounted on two syringe pumps. The core solution sent at a rate of $1 \mathrm{~mL} / \mathrm{h}$, whereas the shell solution was sent at a rate of $3 \mathrm{~mL} / \mathrm{h}$ towards to collector. The electrospinning of the nanofibers was performed at $25^{\circ} \mathrm{C}$ under $18-20 \%$ relative humidity.

\subsection{Characterizations and measurements}

Phase solubility study was performed in aqueous solution according to the previously described method (Higuchi and Connors, 1965). Excess amount of CUR was added into the solutions containing varying amount of HP $\beta C D(0-20 \mathrm{mM})$. The suspensions were stirred overnight at RT and spectrophotometric determination was done at $425 \mathrm{~nm}$ (Varian, Cary 100) after the filtration of the solutions. The measurements were carried out in triplicate and the phase diagram was drawn by plotting the molar concentration of CUR against the molar concentration of HP $\beta C D$ according to the calibration curve.

CUR/HP $\beta C D$-IC was formed according to the co-precipitation method at 1:2 molar ratio (CUR:HP $\beta C D$ ) and the final molar ratio of CUR/HP $\beta C D-I C$ was confirmed by proton nuclear magnetic resonance $\left({ }^{1} \mathrm{H}\right.$ NMR) measurement. First of all, HP $\beta C D$ was dissolved in aqueous solution; then CUR was added and the solution was stirred for $12 \mathrm{~h}$ at RT. Finally, the solution was filtrated after keeping it in refrigerator for $6 \mathrm{~h}$ and dried in hood for 2 days. ${ }^{1} \mathrm{H}$ NMR spectra of CUR, HP $\beta C D$, and CUR/HP $\beta C D-I C$ dissolved in DMSO-d6 were taken on Bruker DPX-400. The assignment of protons of CUR and HP $\beta C D$ are depicted in Fig. S1. Then, the molar ratio of CUR and HP $\beta C D$ in CUR/HP $\beta C D-I C$ was calculated by using the integration of the chemical shifts $(\delta)$ given in parts per million (ppm) calculated via Mestrenova software.

The morphological characterization of cCUR/HP $3 C D-I C-s P L A-$ NF was performed by transmission electron microscopy (TEM, Tecnai G2 F30), confocal laser scanning microscopy (CLSM, Zeiss LSM 510), and scanning electron microscopy (SEM, FEI - Quanta 200 FEG). The SEM imaging of PLA-CUR-NF as a control sample was also performed. The nanofiber samples were sputtered with $5 \mathrm{~nm}$ of $\mathrm{Au} / \mathrm{Pd}$ (PECS-682) to avoid charging problem during SEM imaging. The calculation of average fiber diameter (AFD) of the nanofibers was made on SEM images $(n \geq 100)$ and the results are given as average \pm standard deviation. For the proof of core-shell morphology of cCUR/HP $\beta C D-I C-s P L A-N F$, zinc acetate dehydrate was added to the core solution (CUR/HP $\beta C D-I C)$ and core-shell nanofibers were collected on TEM grids. For CLSM imaging, FITC was added to the core solution (CUR/HP $\beta C D-I C$ ) and nanofibers were collected on glass slides.

X-ray diffraction (XRD) was employed to investigate the crystalline structure of CUR (powder), HPBCD (powder), PLA-NF, and cCUR/HPBCD-IC-sPLA-NF. XRD data were recorded using a PANalytical X'Pert powder diffractometer applying $\mathrm{Cu} \mathrm{K}$ radiation in the 2 theta range of $5-30^{\circ}$.

Thermal properties of CUR (powder), HP $\beta C D$ (powder), PLA-NF, PLA-CUR-NF, and cCUR/HP $\beta C D-I C-s P L A-N F$ were examined by thermal gravimetric analysis (TGA, TA Q500, USA). The samples were heated up to $500^{\circ} \mathrm{C}$ at a constant heating rate of $20^{\circ} \mathrm{C} / \mathrm{min}$ under nitrogen atmosphere for TGA measurement.

Nanofibers having equivalent amount of CUR was immersed in $25 \mathrm{~mL}$ of $0.1 \mathrm{M} \mathrm{HCl}$ ( $\mathrm{pH} \mathrm{1)}$ ) PBS (pH 7.4), methanol and methanol: water (1:1) at RT for $120,480,60$, and $600 \mathrm{~min}$, respectively. The cumulative amount of CUR released from PLA-CUR-NF and CCUR/ HP $\beta C D-I C-s P L A-N F$ was investigated via UV spectroscopy for $0.1 \mathrm{M}$ $\mathrm{HCl}(\mathrm{pH} 1)$ and $\mathrm{PBS}(\mathrm{pH}$ 7.4) and high performance liquid chromatography (HPLC, Agilient, 1200 series) equipped with VWD UV detector $(425 \mathrm{~nm})$ for methanol and methanol:water (1:1). $0.5 \mathrm{~mL}$ of solution was withdrawn at predetermined time intervals and an equal amount of fresh medium was refilled for HPLC measurement. The separation was accomplished by C18 column (Inertisil, column dimension: $4.6 \mathrm{~mm} 50 \mathrm{~mm}$, particle size: $5 \mu \mathrm{m}$ ) operating at $1 \mathrm{~mL} / \mathrm{min}$ using methanol as an eluent. The calibration curves were obtained to convert absorbance and area values obtained from UV spectroscopy and HPLC to concentration (ppm). The experiments were performed in triplicate and the results were reported as average \pm standard deviation. The morphology of nanofibers was also evaluated after immersion of nanofibers into $0.1 \mathrm{M} \mathrm{HCl}(\mathrm{pH} 1)$ and PBS (pH 7.4).

According to 2,2-diphenyl-1-picrylhydrazyl (DPPH) radical scavenging assay, antioxidant activity of PLA-CUR-NF and cCUR/ HP $\beta C D-I C-s P L A-N F$ were tested depending on concentration and time. Concentration dependent antioxidant activity tests were done by immersing nanofibers having equivalent amount of CUR in methanol (for $60 \mathrm{~min}$ ) and methanol:water (1:1) (for $600 \mathrm{~min}$ ) as decided from release tests. The dilution of the solutions were done in methanol and methanol:water (1:1), respectively. Then, $1 \mathrm{~mL}$ of those solutions were mixed with $2 \mathrm{~mL}$ of $10^{-4} \mathrm{DPPH}$ prepared in methanol. After incubation of the solutions in dark at RT for $60 \mathrm{~min}$, absorbance of the solutions was determined by UV spectroscopy (Varian, Cary 100) at $517 \mathrm{~nm}$. In order to calculate antioxidant activity (\%), the absorbance of DPPH was defined as $100 \%$ and the antioxidant activity (\%) was calculated based on the following equation: 
Antioxidant activity $(\%)=\left(\mathrm{A}_{\text {control }}-\mathrm{A}_{\text {sample }}\right) / \mathrm{A}_{\text {control }} \times 100$

where $A_{\text {control }}$ and $A_{\text {sample }}$ represent the absorbance values of control DPPH solution and DPPH solution with nanofibers, respectively. Efficient concentration (EC50) was defined as the amount of antioxidant molecule necessary to decrease DPPH concentration by $50 \%$ (Brand-Williams et al., 1995). For time dependent tests, nanofibers with equivalent amount of CUR were immersed in methanol (for $60 \mathrm{~min}$ ) and methanol:water (1:1) (for $600 \mathrm{~min}$ ) and $1 \mathrm{~mL}$ of those solutions were mixed with $2 \mathrm{~mL}$ of $10^{-4}$ DPPH prepared in methanol. Then, the mixtures were incubated in dark at RT for $60 \mathrm{~min}$. The absorbance of the solutions was measured by UV spectroscopy at $517 \mathrm{~nm}$.

\section{Results and discussion}

\subsection{Phase solubility studies}

Phase solubility diagram for CUR:HP $\beta C D$ system is shown in Fig. 2. The inset photographs given in Fig. 2 presented the change of transparent color of the solution to yellow as the concentration of dissolved CUR increased. Solubility study was performed with increasing amount of $C D$ in aqueous solution at RT. The solubility of CUR enhanced linearly up to $16 \mathrm{mM}$ of $\mathrm{HP} \beta C D$, beyond that point the curve deviates in a positive direction from linearity. So, the solubility curve of CUR:HP $\beta C D$ system is classified as $A_{p}$ type (Brewster and Loftsson, 2007; Takahashi et al., 2012). A type phase solubility diagram suggests the formation of higher order complexes with respect to $C D$ at higher concentration of HP $\beta C D$ (CUR:HP $\beta C D, 1:>1$ ) as well (Brewster and Loftsson, 2007; Takahashi et al., 2012). Therefore, this result is in agreement with the initially used molar ratio which is $1: 2$ (CUR:HP $\beta C D$ ) and this is also confirmed with the proton nuclear magnetic resonance $\left({ }^{1} \mathrm{H}\right.$ NMR) result as discussed in detail below.

\subsection{The molar ratio of inclusion complex}

The molar ratio of $1: 2$ (CUR:HP $\beta C D$ ) was used for the preparation of inclusion complex between the guest molecule (CUR) and the host molecule (HP $\beta C D$ ). Once the CUR/HP $\beta C D-I C$ is formed, we checked the true molar ratio of CUR:HP $\beta C D$ in the sample by ${ }^{1} \mathrm{H}$ NMR (Fig. S1 in Supplementary material). For the analyses, CUR, HP $\beta C D$, and CUR/HP $\beta C D$-IC was dissolved in DMSO-d6 and then ${ }^{1} \mathrm{H}$ NMR spectra were recorded. The assignment of protons of CUR and HP $\beta C D$ are depicted in Fig. S1 in Supplementary material. The molar ratio of CUR to HP $\beta C D$ was calculated as $1: 2$ by taking the integration of CUR peak at $6 \mathrm{ppm}$ and $H P \beta C D$ peak at $1 \mathrm{ppm}$. Therefore, it was concluded that the

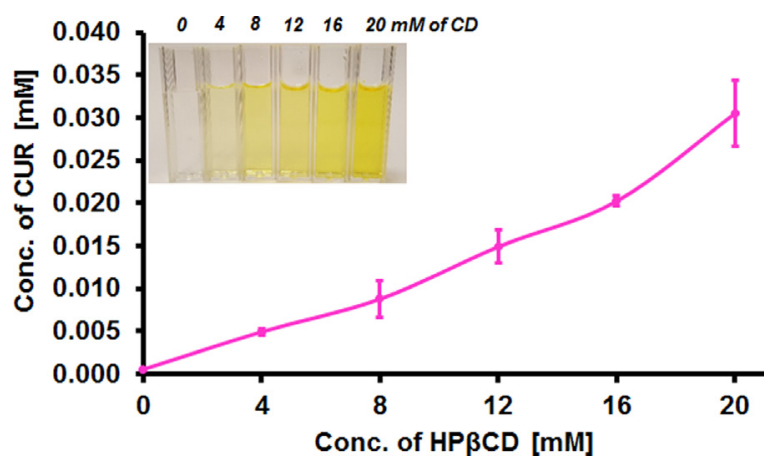

Fig. 2. Phase solubility diagram of CUR/HP $\beta C D$ system in water. Inset photographs show the change of the solution color with increasing HP $\beta C D$ concentration $(0-$ $20 \mathrm{mM}$ ). initial amount of CUR and HP $\beta C D$ (1:2) was preserved perfectly after the inclusion complexation process.

\subsection{Morphology analyses of nanofibers}

Transmission electron microscopy (TEM) and confocal laser scanning microscopy (CLSM) images of cCUR/HPßCD-IC-sPLA-NF are shown in Fig. 3a and 3b. Both of the images confirmed the coreshell structure of nanofibers. The morphology of PLA-CUR-NF and cCUR/HP $\beta C D$-IC-sPLA-NF was further examined by scanning electron microscopy (SEM) Fig. 4a-b. AFD of nanofibers were determined as $780 \pm 375 \mathrm{~nm}$ and $695 \pm 280 \mathrm{~nm}$, respectively. As seen from the photographs given in Fig. $4 c-d$, both of the nanofibers have yellow color but the shade of the nanofibers is obviously different from each other. The reason of the pale yellow color of cCUR/HP $\beta C D-I C-s P L A-N F$ is because the CUR/HP $\beta C D-I C$ is covered with the PLA layer as a shell in this sample.

\subsection{Crystalline structure of the nanofibers}

The crystalline structure of CUR, HPßCD, PLA-NF, and CCUR/ HP $\beta C D-I C-s P L A-N F$ were investigated via X-ray diffraction (XRD) (Fig. S2). CUR is a crystalline molecule, whereas HP $\beta C D$ is amorphous molecule as seen from the diffraction patterns. As seen from the diffraction pattern, cCUR/HPßCD-IC-sPLA-NF did not show crystalline peaks of CUR. The CUR/HP $\beta C D-I C$ is loaded in the fiber matrix as a core and it is expected that CUR would preserve its crystalline phase if there is no true inclusion complexation with the HP $\beta C D$. It is well known that once inclusion complex is formed, guest molecules are separated from each other by the cavity of the CD molecule and therefore cannot form crystals (Giordano et al., 2001). Here, the absence of crystalline peaks of CUR for cCUR/ HP $\beta C D-I C-s P L A-N F$ sample suggested that the true inclusion complexation between CUR and HPßCD was preserved even after the electrospinning process.

\subsection{Thermal analyses of nanofibers}

Thermal gravimetric analysis (TGA) of CUR, HP $\beta C D$, PLA-NF, PLA-CUR-NF, and cCUR/HPBCD-IC-sPLA-NF are given in Fig. S3. The thermal degradation of CUR started at around $200^{\circ} \mathrm{C}$. Native $\mathrm{HP} \beta C D$ exhibited its main degradation with a weight loss in the temperature range from $290^{\circ} \mathrm{C}$ to $425^{\circ} \mathrm{C}$. The weight loss of PLA$\mathrm{NF}$ is observed between $200^{\circ} \mathrm{C}$ and $375^{\circ} \mathrm{C}$ and corresponds to the degradation of PLA. The weight loss in TGA thermograms of PLACUR-NF and cCUR/HPßCD-IC-sPLA-NF is seen between $215-375^{\circ} \mathrm{C}$ and $215-425^{\circ} \mathrm{C}$, respectively. So, the thermal stability of CUR slightly improved in PLA-CUR-NF and cCUR/HPßCD-IC-SPLA-NF. Because of the overlapping in the thermal degradation of CUR, PLA, and $H P \beta C D$, the amount of CUR in nanofibers could not be determined from TGA data.
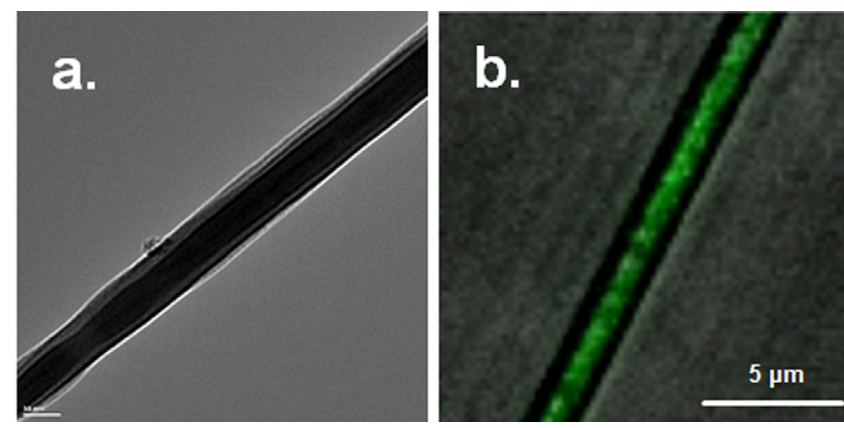

Fig. 3. (a) TEM and (b) CLSM image of $c C U R / H P \beta C D-I C-s P L A-N F$. 

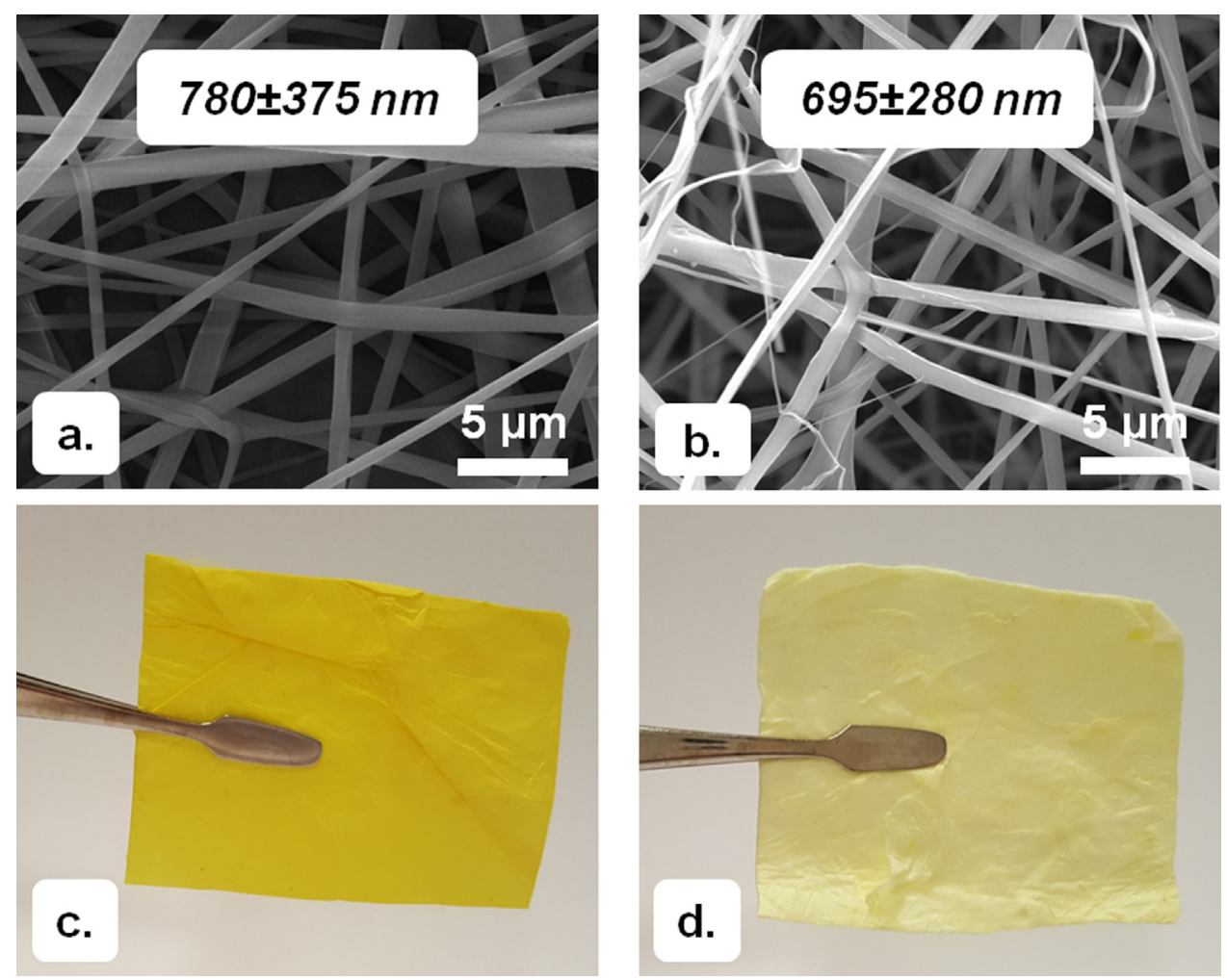

Fig. 4. SEM image of electrospun nanofibers obtained from the solutions of (a) PLA-CUR and (b) cCUR/HPßCD-IC-sPLA; the photographs of (c) PLA-CUR-NF and (d) cCUR/ HPBCD-IC-SPLA-NF.

\section{6. in vitro release study}

The $\mathrm{pH}$ dependent release of CUR from PLA-CUR-NF and cCUR/ HPßCD-IC-sPLA-NF were investigated in $0.1 \mathrm{M} \mathrm{HCl}(\mathrm{pH} 1$, simulated gastric fluid) and $\mathrm{PBS}(\mathrm{pH} 7.4$, simulated intestinal fluid) at RT (Fig. 5a-b). Higher amount of CUR released from both of the nanofibers at $\mathrm{pH} 1$ compared to $\mathrm{pH}$ 7.4. Since CUR is known to exist in cationic, neutral or anionic forms depending on $\mathrm{pH}$ and these forms influence the solubility of CUR. Hence, when pH is acidic, CUR releases readily from the nanofibers due to increased solubility when it is in cationic form (Massaro et al., 2016). Moreover, the rate of release from cCUR/HPßCD-IC-sPLA-NF was slower compared to PLA-CUR-NF at the initial step at both $\mathrm{pH} 1$ and $\mathrm{pH} 7.4$ owing to shell structure in CCUR/HP $\beta C D-I C-s P L A-N F$. On the other hand, CUR released from cCUR/HPßCD-IC-sPLA-NF was much more in total than PLA-CUR-NF at $\mathrm{pH} 1$ and $\mathrm{pH} 7.4$ most probably due to the solubility enhancement of CUR by HP $\beta C D$ inclusion complexation as shown in Fig. 2. After in vitro release test, possibility of fiber matrix degradation was investigated by the morphology change in SEM images (Fig. S4 in Supplementary material). If nanofiber matrix undergoes degradation overtime, erosion based release mechanism is anticipated. However, SEM images clearly showed that PLA-CUR-NF and cCUR/HPßCD-ICsPLA-NF samples preserved their fibrous structure during the release period, and therefore, in our case the release mechanism is diffusion based.

The release of CUR was evaluated in methanol and methanol: water (1:1) as well (Fig. 6a-b). PLA-CUR-NF released much more CUR in methanol; whereas cCUR/HP $\beta C D-I C-s P L A-N F$ released slightly more amount of CUR in methanol:water (1:1) comparative to its counterpart. This is likely due to the high solubility of CUR in methanol when it is in free form and enhanced water solubility of CUR in methanol:water $(1: 1)$ when it is formed an IC with HPßCD.
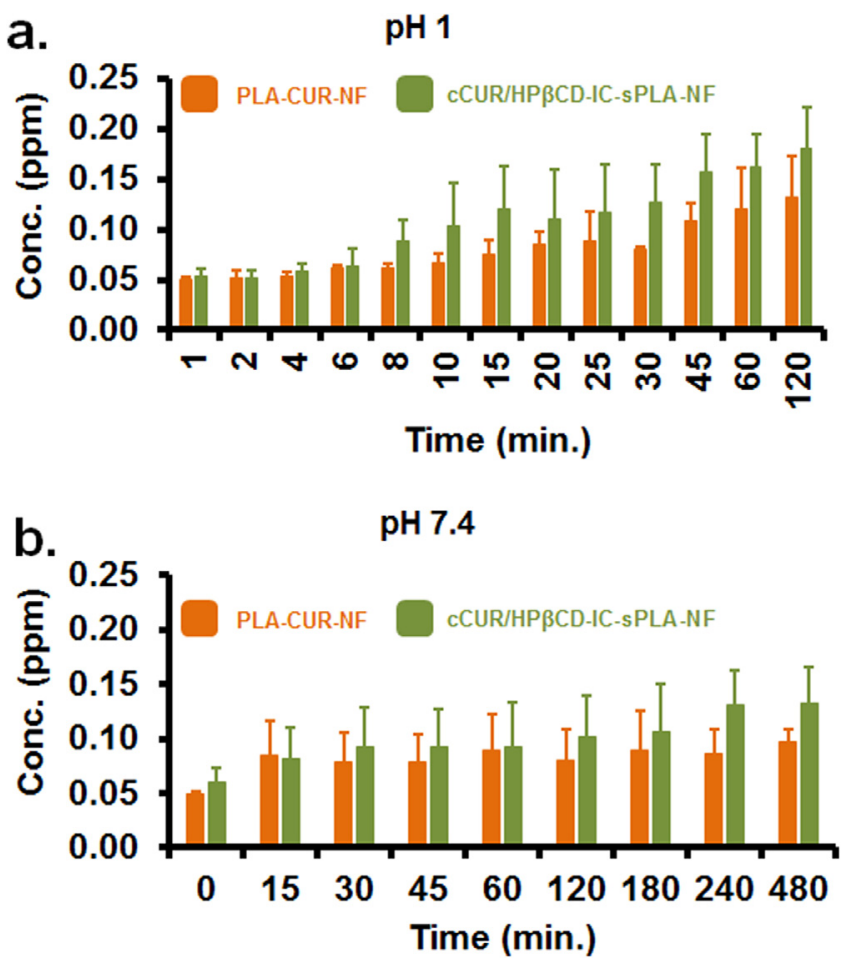

Fig. 5. The cumulative release of CUR from PLA-CUR-NF and CCUR/HPßCD-IC-sPLA$\mathrm{NF}$ into (a) $\mathrm{pH} 1$ and (b) $\mathrm{pH} 7.4(\mathrm{n}=3)$. The error bars in the figure represent the standard deviation (SD). 

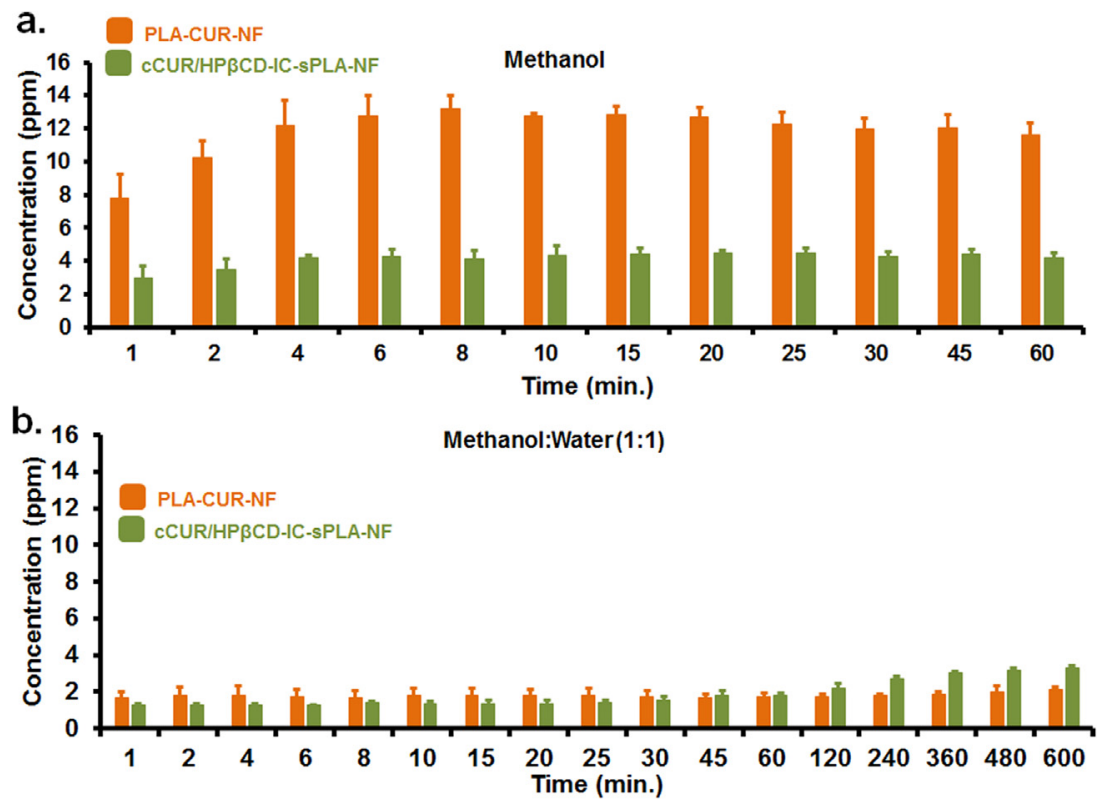

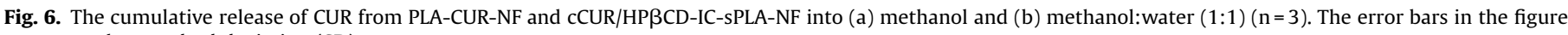
represent the standard deviation (SD).

\subsection{Antioxidant activity}

The reduction of chronic diseases, DNA damage, mutagenesis, carcinogenesis, and inhibition pathogenic bacterial growth is mostly related with the free radical scavenging ability of antioxidant compounds (Ak and Gulcin, 2008). Antioxidant activity of molecules is often due to the presence of the phenolic hydrogen in their structure, but since CUR's phenolic hydrogen atoms are intramolecularly hydrogen-bonded to the methoxy groups, hydrogen abstraction from phenolic ring of CUR is difficult (Gulcin, 2012). However, abstraction of hydrogen from the carbon atom which is in the heptadienone linkage between the two methoxyphenol rings is relatively easier and this abstraction is the main reason of antioxidant activity of CUR (Gulcin, 2012).

Antioxidant activity of PLA-CUR-NF and cCUR/HP $\beta C D-I C-s P L A-$ $\mathrm{NF}$ was tested by 2,2-diphenyl-1-picrylhydrazyl (DPPH) with respect to concentration and time firstly by extracting CUR from nanofibers in methanol or methanol:water (1:1) (Figs. 7 and 8). For methanol, PLA-CUR-NF and cCUR/HPßCD-IC-sPLA-NF has $44 \pm 2-$ $95 \pm 0 \%$ and $34 \pm 1-94 \pm 3 \%$ antioxidant activity in the concentration range of $5-160 \mathrm{ppm}$, respectively (Fig. 7a). According to concentration dependent test made in methanol, efficient concentration 50 (EC50) was determined between 5 and 10 ppm and 20$40 \mathrm{ppm}$ for PLA-CUR-NF and cCUR/HPßCD-IC-sPLA-NF, respectively. Lower EC50 shows higher free radical scavenging capability of PLA-CUR-NF. This result is also consistent with the release study made in methanol in which PLA-CUR-NF released higher amount of CUR compared to cCUR/HP $\beta C D$-IC-sPLA-NF. The visual investigation of the resulting solutions also shows the coherence with the calculated antioxidant activities (Fig. 7b). For instance, cCUR/ HP $\beta C D$-IC-sPLA-NF exhibited $34 \pm 1 \%$ of antioxidant activity and the color of the solution was purple; when $94 \pm 3 \%$ of antioxidant activity was seen, the color of the solution became yellow.

Time dependent antioxidant activity of nanofibers from which methanol was used to extract CUR was evaluated for $60 \mathrm{~min}$ (Fig. 7c). PLA-CUR-NF has reached its maximum antioxidant activity in $2 \mathrm{~min}$ ( $93 \pm 1 \%$ ); whereas cCUR/HPßCD-IC-sPLA-NF shows its maximum antioxidant activity $(84 \pm 3 \%)$ in $15 \mathrm{~min}$. The slow antioxidant activity of cCUR/HP $\beta C D-I C-s P L A-N F$ as compared to PLA-CUR-NF is due to the presence of an additional hydrophobic barrier (shell) delaying the access of water and dissolution of CUR.

The antioxidant activity of PLA-CUR-NF and cCUR/HPßCD-ICsPLA-NF extracted using methanol:water (1:1) was calculated as $44 \pm 3-89 \pm 1 \%$ and $39 \pm 1-92 \pm 3 \%$ in the range of $5-160 \mathrm{ppm}$,

a.

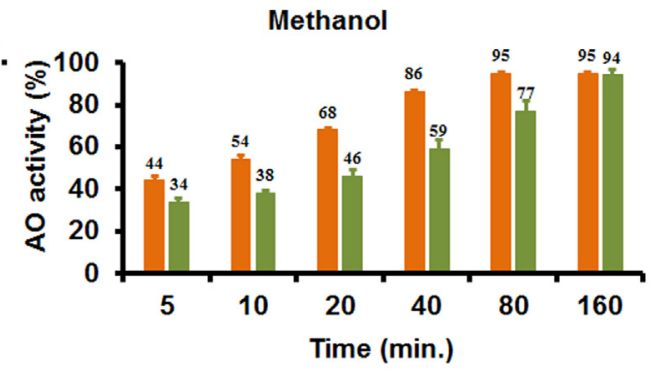

b.

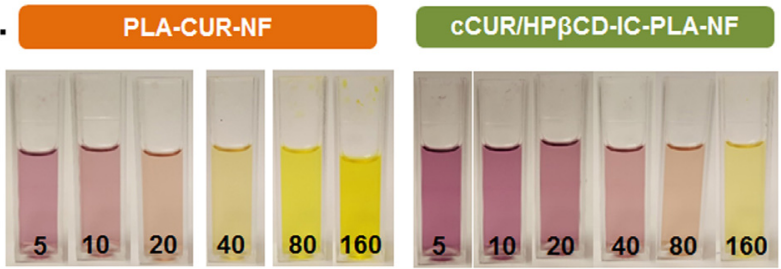

c.

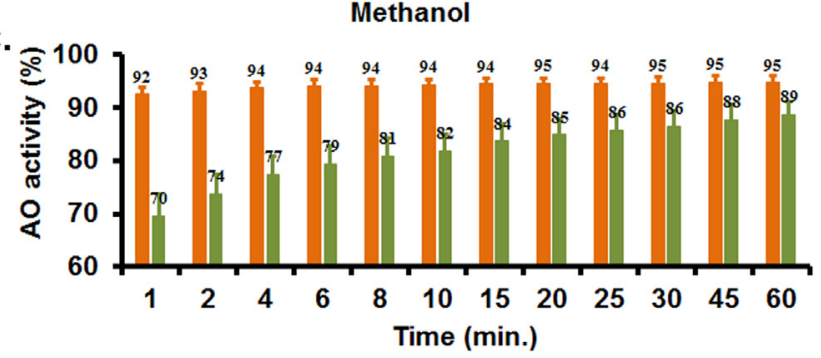

Fig. 7. (a) Concentration dependent antioxidant activity of PLA-CUR-NF and cCUR/ HPßCD-IC-sPLA-NF (methanol), (b) the photographs of the solutions with respect to concentration; (c) time dependent antioxidant activity of PLA-CUR-NF and cCUR/ HPßCD-IC-sPLA-NF (methanol). 
a.

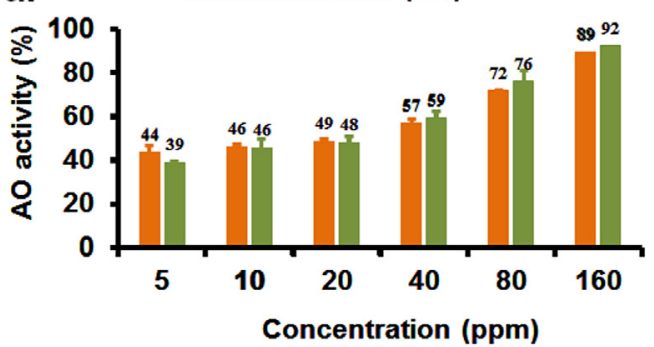

b.
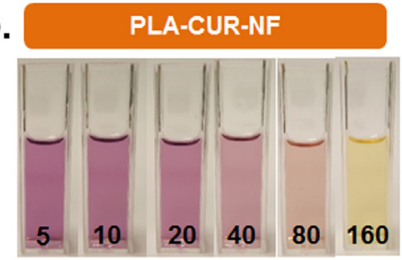

Methanol:Water (1:1)

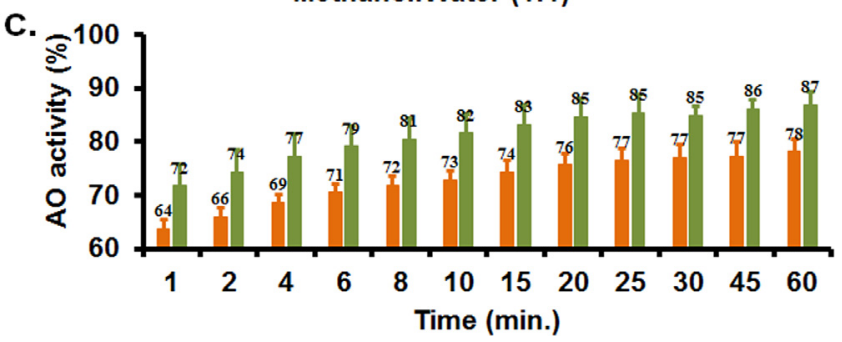

Fig. 8. (a) Concentration dependent antioxidant activity of PLA-CUR-NF and cCUR/ HPßCD-IC-sPLA-NF (methanol:water, 1:1), (b) the photographs of the solutions with respect to concentration; (c) time dependent antioxidant activity of PLA-CURNF and cCUR/HPßCD-IC-sPLA-NF (methanol:water, 1:1).

respectively (Fig. 8a). EC50 for both PLA-CUR-NF and cCUR/ HPBCD-IC-SPLA-NF was decided to be slightly higher than $20 \mathrm{ppm}$. So, cCUR/HPßCD-IC-sPLA-NF exhibited quite similar antioxidant capability with PLA-CUR-NF. This result is compatible with the release study; thus, when the solubility of CUR was enhanced by complexation, cCUR/HPßCD-IC-sPLA-NF exhibited antioxidant activity quite well. The photographs of each solution show the change of purple to yellow color with the increasing concentration of CUR (Fig. 8b).

Time dependent antioxidant activity of PLA-CUR-NF and cCUR/ HP $\beta C D$-IC-sPLA-NF was measured for $60 \mathrm{~min}$ and antioxidant activities were decided as $78 \pm 2 \%$ and $87 \pm 2 \%$ for PLA-CUR-NF and whereas cCUR/HP $\beta C D-I C-S P L A-N F$, respectively (Fig. 8c). The maximum antioxidant activity of PLA-CUR-NF and cCUR/HP $\beta C D-$ IC-sPLA-NF were obtained in $15 \mathrm{~min}(74 \pm 2 \%)$ and $8 \mathrm{~min}(81 \pm 4 \%)$, respectively. This result indicated relatively quick antioxidant activity of cCUR/HP $\beta C D-I C-$ SPLA-NF in methanol:water $(1: 1)$ in comparison with methanol owing to the greater solubility of CUR in water based system.

\section{Conclusions}

Core-shell nanofibers were produced using cyclodextrininclusion complex (CD-IC) of a model hydrophobic drug (curcumin, CUR) in the core and polylactic acid (PLA) in the shell (cCUR/ HP $\beta C D-I C-s P L A-N F$ ) by electrospinning. CUR released from cCUR/ HP $\beta C D-I C-s P L A-N F$ was much more than PLA-CUR-NF at pH 1 and pH 7.4 most probably due to the solubility enhancement as shown in phase solubility diagram. In addition, due to the presence of a shell layer CUR released slower from cCUR/HP $\beta C D-I C-s P L A-N F$ as compared to PLA-CUR-NF. Efficient concentration 50 (EC50) was lower for cCUR/HPßCD-IC-sPLA-NF in methanol:water than in methanol since inclusion complexation of CUR and HP $\beta C D$ improves the solubility of CUR in aqueous solution. The relatively slow antioxidant activity of cCUR/HP $\beta C D-I C-s P L A-N F$ in methanol is likely due to the additional polymeric barrier (shell) delaying the access of water; whereas slightly quick antioxidant activity is associated with the high solubility of CUR/HP $\beta C D-I C$ in methanol: water $(1: 1)$. In short, drug delivery systems based on core-shell nanofiber structure in which hydrophobic drugs are placed in the core structure in the form of inclusion complex with cyclodextrins could provide slow release as well as high water solubility for such hydrophobic drugs.

\section{Acknowledgements}

This work was supported by The Scientific and Technological Research Council of Turkey (TUBITAK)-Turkey (Project \# 111M459). Dr. Uyar also acknowledges The Turkish Academy of SciencesOutstanding Young Scientists Award Program (TUBA-GEBIP)Turkey for the partial support. Z. Aytac thanks to TUBITAK-BIDEB (2211-C) and TUBITAK (project \# 111M459) for the PhD scholarship. We express our special thanks to Dr. Anitha Senthamizhan for her help in the confocal laser scanning microscopy imaging.

\section{Appendix A. Supplementary data}

Supplementary data associated with this article can be found, in the online version, at http://dx.doi.org/10.1016/j. ijpharm.2016.12.061.

\section{References}

Agarwal, S., Wendorff, J.H., Greiner, A., 2008. Use of electrospinning technique for biomedical applications. Polymer 49 (26), 5603-5621.

Aggarwal, B.B., Kumar, A., Bharti, A.C., 2003. Anticancer potential of curcumin: preclinical and clinical studies. Anticancer Res. 23 (1A), 363-398.

Ak, T., Gulcin, I., 2008. Antioxidant and radical scavenging properties of curcumin. Chem. Biol. Interact. 174 (1), 27-37.

Aytac, Z., Uyar, T., 2016. Antioxidant activity and photostability of $\alpha$-tocopherol/ $\beta$-cyclodextrin inclusion complex encapsulated electrospun polycaprolactone nanofibers. Eur. Polym. J. 79, 140-149.

Aytac, Z., Dogan, S.Y., Tekinay, T., Uyar, T., 2014. Release and antibacterial activity of allyl isothiocyanate/ $\beta$-cyclodextrin complex encapsulated in electrospun nanofibers. Colloids Surf. B: Biointerfaces 120, 125-131.

Aytac, Z., Sen, H.S., Durgun, E., Uyar, T., 2015. Sulfisoxazole/cyclodextrin inclusion complex incorporated in electrospun hydroxypropyl cellulose nanofibers as drug delivery system. Colloids Surf. B: Biointerfaces 128, 331-338.

Aytac, Z., Kusku, S.I., Durgun, E., Uyar, T., 2016a. Encapsulation of gallic acid/ cyclodextrin inclusion complex in electrospun polylactic acid nanofibers: release behavior and antioxidant activity of gallic acid. Mater. Sci. Eng. C 63 , 231-239.

Aytac, Z., Kusku, S.I., Durgun, E., Uyar, T., 2016b. Quercetin/ $\beta$-cyclodextrin inclusion complex embedded nanofibres: slow release and high solubility. Food Chem. $197,864-871$.

Baglole, K.N., Boland, P.G., Wagner, B.D., 2005. Fluorescence enhancement of curcumin upon inclusion into parent and modified cyclodextrins. J. Photochem. Photobiol. A: Chem. 173 (3), 230-237.

Bansal, S.S., Kausar, H., Aqil, F., Jeyabalan, J., Vadhanam, M.V., Gupta, R.C., Ravoori, S., 2011. Curcumin implants for continuous systemic delivery: safety and biocompatibility. Drug Deliv. Transl. Res. 1 (4), 332-341.

Brand-Williams, W., Cuvelier, M.E., Berset, C.L.W.T., 1995. Use of a free radical method to evaluate antioxidant activity. LWT-Food Sci. Technol. 28 (1), 25-30.

Brewster, M.E., Loftsson, T., 2007. Cyclodextrins as pharmaceutical solubilizers. Adv. Drug Deliv. Rev. 59 (7), 645-666.

Del Valle, E.M., 2004. Cyclodextrins and their uses: a review. Process Biochem. 39 (9), 1033-1046.

Giordano, F., Novak, C., Moyano, J.R., 2001. Thermal analysis of cyclodextrins and their inclusion compounds. Thermochim. Acta 380 (2), 123-151.

Greiner, A., Wendorff, J.H., 2007. Electrospinning: a fascinating method for the preparation of ultrathin fibers. Angew. Chem. Int. Ed. 46 (30), 5670-5703.

Gulcin, I., 2012. Antioxidant activity of food constituents: an overview. Arch. Toxicol. 86 (3), 345-391.

Guo, G., Fu, S., Zhou, L., Liang, H., Fan, M., Luo, F., Qian, Z., Wei, Y., 2011. Preparation of curcumin loaded poly ( $\varepsilon$-caprolactone)-poly (ethylene glycol)-poly ( $\varepsilon$-caprolactone) nanofibers and their in vitro antitumor activity against Glioma 9L cells. Nanoscale 3 (9), 3825-3832. 
Hedges, A.R., 1998. Industrial applications of cyclodextrins. Chem. Rev. 98 (5), 2035 2044.

Higuchi, T.K., Connors, A., 1965. Phase-solubility techniques. Adv. Anal. Chem. Instrum. 4, 117-212.

Jiang, H., Hu, Y., Li, Y., Zhao, P., Zhu, K., Chen, W., 2005. A facile technique to prepare biodegradable coaxial electrospun nanofibers for controlled release of bioactive agents. J. Control. Release 108 (2), 237-243.

Jiang, H., Wang, L., Zhu, K., 2014. Coaxial electrospinning for encapsulation and controlled release of fragile water-soluble bioactive agents. J. Control. Release 193, 296-303.

Kayaci, F., Uyar, T., 2012. Encapsulation of vanillin/cyclodextrin inclusion complex in electrospun polyvinyl alcohol (PVA) nanowebs: prolonged shelf-life and high temperature stability of vanillin. Food Chem. 133 (3), 641-649.

Kayaci, F., Ertas, Y., Uyar, T., 2013a. Enhanced thermal stability of eugenol by cyclodextrin inclusion complex encapsulated in electrospun polymeric nanofibers. J. Agric. Food Chem. 61 (34), 8156-8165.

Kayaci, F., Umu, O.C., Tekinay, T., Uyar, T., 2013b. Antibacterial electrospun poly (lactic acid) (PLA) nanofibrous webs incorporating triclosan/cyclodextrin inclusion complexes. J. Agric. Food Chem. 61 (16), 3901-3908.

Kayaci, F., Sen, H.S., Durgun, E., Uyar, T., 2014. Functional electrospun polymeric nanofibers incorporating geraniol-cyclodextrin inclusion complexes: high thermal stability and enhanced durability of geraniol. Food Res. Int. 62, 424-431.

Kumar, S.U., Matai, I., Dubey, P., Bhushan, B., Sachdev, A., Gopinath, P., 2014. Differentially cross-linkable core-shell nanofibers for tunable delivery of anticancer drugs: synthesis, characterization and their anticancer efficacy. RSC Adv. 4 (72), 38263-38272.

Llorens, E., Ibañez, H., Del Valle, L.J., Puiggalí, J., 2015. Biocompatibility and drug release behavior of scaffolds prepared by coaxial electrospinning of poly (butylene succinate) and polyethylene glycol. Mater. Sci. Eng. C 49, 472-484.

Massaro, M., Amorati, R., Cavallaro, G., Guernelli, S., Lazzara, G., Milioto, S., Noto, R., Poma, P., Riela, S., 2016. Direct chemical grafted curcumin on halloysite nanotubes as dual-responsive prodrug for pharmacological applications. Colloids Surf. B: Biointerfaces 140, 505-513.

Ramakrishna, S., Fujihara, K., Teo, W.E., Lim, T.C., Ma, Z., 2005. An Introduction to Electrospinning and Nanofibers, vol. 90. World Scientific, Singapore.

Sampath, M., Lakra, R., Korrapati, P., Sengottuvelan, B., 2014. Curcumin loaded poly (lactic-co-glycolic) acid nanofiber for the treatment of carcinoma. Colloids Surf. B: Biointerfaces 117, 128-134.

Sedghi, R., Shaabani, A., 2016. Electrospun biocompatible core/shell polymer-free core structure nanofibers with superior antimicrobial potency against multi drug resistance organisms. Polymer 101, 151-157.
Siviero, A., Gallo, E., Maggini, V., Gori, L., Mugelli, A., Firenzuoli, F., Vannacci, A., 2015. Curcumin, a golden spice with a low bioavailability. J. Herb. Med. 5 (2), 57-70.

Sun, Z., Zussman, E., Yarin, A.L., Wendorff, J.H., Greiner, A., 2003. Compound coreshell polymer nanofibers by co-electrospinning. Adv. Mater. 15 (22),1929-1932.

Sun, X.Z., Williams, G.R., Hou, X.X., Zhu, L.M., 2013. Electrospun curcumin-loaded fibers with potential biomedical applications. Carbohydr. Polym. 94 (1), 147153.

Sun, Y., Du, L., Liu, Y., Li, X., Li, M., Jin, Y., Qian, X., 2014. Transdermal delivery of the in situ hydrogels of curcumin and its inclusion complexes of hydroxypropyl$\beta$-cyclodextrin for melanoma treatment. Int. J. Pharm. 469 (1), 31-39.

Suwantong, O., Opanasopit, P., Ruktanonchai, U., Supaphol, P., 2007. Electrospun cellulose acetate fiber mats containing curcumin and release characteristic of the herbal substance. Polymer 48 (26), 7546-7557.

Szejtli, J., 1998. Introduction and general overview of cyclodextrin chemistry. Chem. Rev. 98 (5), 1743-1754.

Takahashi, A.I., Veiga, F.J.B., Ferraz, H.G., 2012. Literature review of cyclodextrins inclusion complexes characterization - Part I: Phase solubility diagram, dissolution and scanning electron microscopy. Int. J. Pharm. Sci. Rev. Res. 12 (1), $1-6$.

Uyar, T., Hacaloglu, J., Besenbacher, F., 2009a. Electrospun polystyrene fibers containing high temperature stable volatile fragrance/flavor facilitated by cyclodextrin inclusion complexes. React. Funct. Polym. 69 (3), 145-150.

Uyar, T., Nur, Y., Hacaloglu, J., Besenbacher, F., 2009b. Electrospinning of functional poly (methyl methacrylate) nanofibers containing cyclodextrin-menthol inclusion complexes. Nanotechnology 20 (12), 125703.

Uyar, T., Hacaloglu, J., Besenbacher, F., 2011. Electrospun polyethylene oxide (PEO) nanofibers containing cyclodextrin inclusion complex. J. Nanosci. Nanotechnol. 11 (5), 3949-3958.

Wang, X., Yu, D.G., Li, X.Y., Bligh, S.A., Williams, G.R., 2015. Electrospun medicated shellac nanofibers for colon-targeted drug delivery. Int. J. Pharm. 490 (1), 384 390.

Wendorff, J.H., Agarwal, S., Greiner, A., 2012. Electrospinning: Materials, Processing, and Applications. John Wiley \& Sons, Weinheim.

Yadav, V.R., Suresh, S., Devi, K., Yadav, S., 2009. Effect of cyclodextrin complexation of curcumin on its solubility and antiangiogenic and anti-inflammatory activity in rat colitis model. AAPS PharmSciTech. 10 (3), 752-762.

Yallapu, M.M., Nagesh, P.K.B., Jaggi, M., Chauhan, S.C., 2015. Therapeutic applications of curcumin nanoformulations. AAPS J. 17 (6), 1341-1356. 